\title{
Management of Hypertension in Diabetic Nephropathy: How Low Should We Go?
}

\author{
Hillel Sternlicht George L. Bakris \\ Department of Medicine, Section of Endocrinology, Diabetes and Metabolism, ASH Comprehensive Hypertension \\ Center, The University of Chicago Medicine, Chicago, IL, USA
}

\section{Key Words}

Hypertension · Nephropathy · Diabetes

\begin{abstract}
Hypertension is a frequent comorbidity often following the development of diabetic nephropathy among individuals with type 1 diabetes and affecting most patients with type 2 diabetes at the time of diagnosis. Multiple prospective randomized placebo-controlled trials demonstrate that tight blood pressure control among patients with diabetic nephropathy reduces the rates of macrovascular and microvascular complications. While randomized trials exist and support a blood pressure goal of $<140 / 90 \mathrm{~mm} \mathrm{Hg}$ for patients with nondiabetic kidney disease, there are no prospective data regarding a specific blood pressure goal on progression of diabetic nephropathy. Retrospective data analyses from trials show a linear relationship between either baseline or achieved study blood pressure and progression of nephropathy. Very high albuminuria is a hallmark of diabetic nephropathy with reductions by either angiotensin converting enzyme inhibitors (ACEi) or angiotensin receptor blocker (ARB) monotherapy associated with slowed nephropathy progression. However, combination antihypertensive therapy, while decreasing proteinuria, augments the risk of hyperkalemia, hypotension, and kidney dysfunction. Given the lack of trial data for a BP goal among patients with diabetic nephropathy, prospective trials are needed to define the optimal blood pressure necessary to preserve kidney function.
\end{abstract}

At present, guideline blood pressure goals of less than 140/90 $\mathrm{mm} \mathrm{Hg}$ and the use of ACEi or ARB therapy for those with more than $300 \mathrm{mg}$ of albuminuria are mandated.

(c) 2016 S. Karger AG, Base

\section{Epidemiology of Hypertension in Diabetic Nephropathy}

Hypertension is twice as prevalent in patients with diabetes compared to the general population with mean blood pressure rising by $5-8 \%$ a year in those with overt nephropathy, a condition affecting $35 \%$ of type 1 individuals and $25 \%$ of type 2 patients [1]. The characteristics and natural history of hypertension vary by subtypes of diabetes. Among type 1 patients, the incidence of hypertension rises in tandem with that of albuminuria such that hypertension affects $4 \%$ of normoalbuminuric patients, $25 \%$ of high albuminuric patients, and $80 \%$ of very high albuminuric patients [2]. An in-depth review of the subject is summarized elsewhere [3].

\section{Extra-Renal Benefits of Improved Blood Pressure Control}

The vast majority of randomized controlled trials as well as a subsequent meta-analysis support the extra-renal benefits of tighter blood pressure control among pa-

\section{KARGER}

E-Mail karger@karger.com

www.karger.com/bpu
(C) 2016 S. Karger AG, Basel

0253-5068/16/0413-0139\$39.50/0
George L. Bakris, MD

University of Chicago Medicine

5841 S. Maryland Ave. MC 1027

Chicago, IL 60637 (USA)

E-Mail gbakris@gmail.com 
Table 1. Achieved blood pressure in diabetes trials by outcome

Trial name

(outcome)

Achieved systolic blood pressure, $\mathrm{mm} \mathrm{Hg}$

UKPDS (primary)

ADVANCE (secondary)

ACCOMPLISH (secondary)

ONTARGET (secondary)

VADT (secondary)

INVEST (secondary)

ACCORD (primary)

Intensive: 144 conventional: 154

Intensive and conventional: 145

Overall mean: 133

Overall mean: 140

Intensive: 127 conventional: 125

Intensive: 144 conventional: 149

Intensive: 119 conventional: 133

tients at risk for cardiovascular complications. A summary of the achieved blood pressures from prospective clinical trials evaluating cardiovascular outcomes in patients with diabetes is shown in table 1.

The United Kingdom Prospective Diabetes Study (UKPDS) targeted a blood pressure of less than 150/85 vs. less than $180 / 105 \mathrm{~mm} \mathrm{Hg}$ achieving a $10 / 5 \mathrm{~mm} \mathrm{Hg}$ difference between arms. This reduced the risk of macrovascular and microvascular complications by $24 \%$ with a $32 \%$ reduction in deaths and a $44 \%$ reduction in strokes [4].

The Action in Diabetes and Vascular disease: preterAx and diamicroN-MR Controlled Evaluation (ADVANCE Trial), showed that in those suffering from type 2 diabetes and with a history of major cardiovascular disease, combination therapy with perindopril/indapamide therapy versus placebo resulted in a decrease in blood pressure difference of 5.6/2.2 $\mathrm{mm} \mathrm{Hg}$ from a baseline pressure of $145 / 81 \mathrm{~mm} \mathrm{Hg}$ [5]. This translated into a 9\% relative risk reduction in major macrovascular and microvascular events including a $14 \%$ reduction in all-cause mortality.

The Avoiding Cardiovascular Events through Combination Therapy in Patients Living with Systolic Hypertension (ACCOMPLISH) Trial evaluated combination therapy with benazepril/amlodipine vs. benazepril/hydrochlorothiazide in patients who were at high risk for cardiovascular events [6]. Sixty percent of participants had diabetes. While both groups achieved a blood pressure of $132 / 73 \mathrm{~mm} \mathrm{Hg}$, the angiotensin converting enzyme inhibitors (ACEi)/calcium channel blocker patients had $20 \%$ less cardiovascular events than those treated with the ACEi/diuretic combination.

A 2015 meta-analysis by Emdin including 40 trials and 100,000 patients with diabetes revealed a $13 \%$ reduction in all-cause mortality and an $11 \%$ reduction in the composite endpoint of myocardial infarction, cardiac death, stroke, heart failure, and revascularization per $10 \mathrm{~mm} \mathrm{Hg}$

decrease in systolic blood pressure [7]. Of note, the graded reduction in cardiovascular events with further blood pressure control was limited to those with initial systolic pressures greater than $140 \mathrm{~mm} \mathrm{Hg}$.

Benefits limited to a blood pressure 'sweet spot' of $140 \mathrm{~mm} \mathrm{Hg}$ systolic are consistent with the most recent publications involving post-hoc analysis of the International Verapamil SR-Trandolapril Study (INVEST), and the Veterans Affairs Diabetes Trial (VADT) as well as prospective data from the Action to Control Cardiovascular Risk in Diabetes (ACCORD) Group. Cooper-DeHoff et al. [8] analyzed cardiovascular outcomes from INVEST by achieved blood pressure categories: tight (systolic pressures of less than $130 \mathrm{~mm} \mathrm{Hg}$ ), usual (systolic pressures of 130-140 mm Hg), or uncontrolled (systolic pressures greater than $140 \mathrm{~mm} \mathrm{Hg}$ ) among patients with both diabetes and coronary artery disease. The primary endpoint occurred in $12.5 \%$ of patients in either the tight or standard blood pressure groups but in $20 \%$ of the uncontrolled cohort indicating a lack of effect when blood pressure is lowered below a systolic pressure of $140 \mathrm{~mm} \mathrm{Hg}$.

The ACCORD investigators prospectively evaluated whether achieving a systolic pressure of $120 \mathrm{~mm} \mathrm{Hg}$ as compared to an achieved systolic pressure of $134 \mathrm{~mm} \mathrm{Hg}$ reduced the risk of cardiovascular events among those diagnosed with diabetes at high risk for such events. After 1 year of follow-up, the number of cardiac events was similar among the 2 arms but the rate of adverse events such as electrolyte disarray, elevated creatinine, and hypotension was 3 times more common among those assigned to the lower blood pressure goal [9].

Finally, the VADT study, which prospectively assessed the impact of intensive glucose control on cardiovascular outcomes among veterans with longstanding diabetes and suboptimal glycemic control, retrospectively evaluated blood pressure strata. This analysis demonstrated a hazard ratio of 1.5 for the primary cardiovascular endpoint when either entry or achieved systolic blood pressure was above $140 \mathrm{~mm} \mathrm{Hg} \mathrm{[10].}$

\section{Benefits on Kidney Disease Progression with Blood Pressure Control}

While studies evaluating the impact of blood pressure on CKD progression exist, to date, there have been no prospective studies evaluating specific blood pressure goals on the progression of diabetic nephropathy. Nonetheless, current guidelines generated by various groups from both general medical and nephrology societies gen- 
erally recommend a blood pressure goal of less than 140/90 $\mathrm{mm} \mathrm{Hg}$ and the use of ACEi or angiotensin receptor blockers (ARBs) for slowing progression of nephropathy among those with greater than $300 \mathrm{mg}$ of albuminuria $[11,12]$.

The earliest data on the impact of blood pressure levels on CKD progression comes from individuals participating in the Hypertension Screening and Treatment Program (HSTP) Study in the 1970s. After following hypertensive patients with and without kidney disease and diabetes for nearly 15 years, those with baseline systolic blood pressures between 160 and $179 \mathrm{~mm} \mathrm{Hg}$ were twice as likely to be dialysis dependent. Moreover, those with a pressure greater than $180 \mathrm{~mm} \mathrm{Hg}$ were 5.5 times more likely to progress to end-stage renal disease (ESRD) compared to those with systolic pressures below these values [13].

A post-hoc analysis of the relationship between baseline blood pressure and renal prognosis in subjects for the Reduction of Endpoints in Non-insulin dependent diabetes with the Angiotensin Antagonist Losartan (RENAAL) Study showed similar results [14]. A systolic blood pressure of less than $140 \mathrm{~mm} \mathrm{Hg}$ immediately prior to study entry was associated with a $50 \%$ higher risk for the primary renal endpoint (fig. 1). Those with a systolic blood pressure between 140 and $160 \mathrm{~mm} \mathrm{Hg}$ had a 2.7 -fold higher risk for the renal endpoint. Furthermore, multivariate analysis demonstrated an increased risk of death or ESRD of $6.7 \%$ for every $10 \mathrm{~mm} \mathrm{Hg}$ increase in baseline systolic blood pressure.

Complementing the analysis of the RENAAL data was an analysis by Pohl et al. [15] that retrospectively stratified renal outcomes of patients from the Irbesartan Diabetic Nephropathy Trial (IDNT) by achieved follow-up blood pressures. Patients with an achieved systolic pressure of greater than $149 \mathrm{~mm} \mathrm{Hg}$ were 2.2 times more likely to reach a renal endpoint compared to those with a systolic pressure of less than $134 \mathrm{~mm} \mathrm{Hg}$ (fig. 2). A decrease in achieved systolic pressure of $20 \mathrm{~mm} \mathrm{Hg}$ resulted in a $50 \%$ reduction in the risk of reaching the primary renal endpoint. Of note, in all of the three above-mentioned trials, diastolic pressure was not predictive of the risk of reaching an adverse renal endpoint.

\section{Relationship between Proteinuria Reduction and Time to ESRD Progression}

Reduction in proteinuria with ACEi or ARB therapy, when it occurs, is associated with slowed progression of diabetic nephropathy. This was first established in the

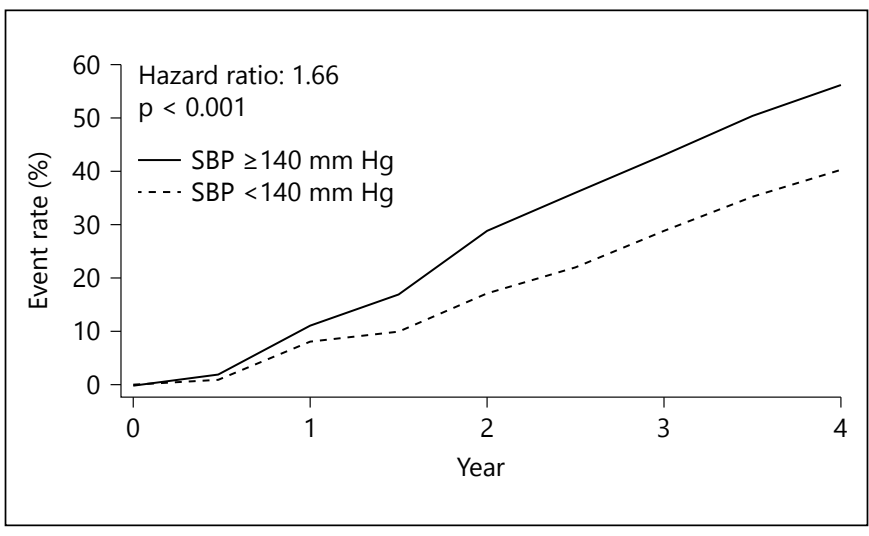

Fig. 1. Event rate for primary renal endpoint by baseline systolic blood pressure (SBP). From Bakris et al. [14].

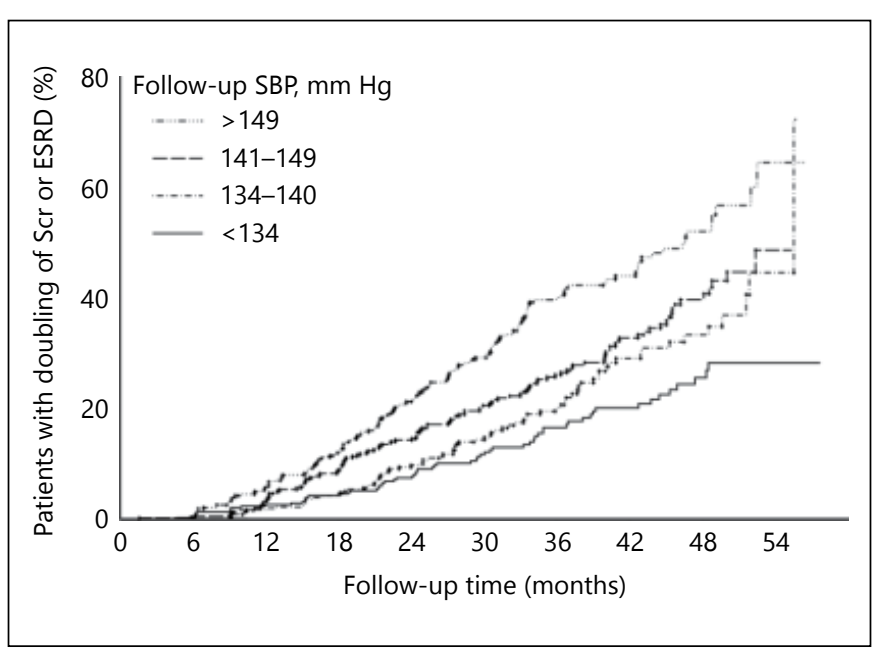

Fig. 2. Kaplan-Meier estimates of rates of doubling of serum creatinine (Scr) or ESRD by achieved blood pressure. From Pohl et al. [15].

Captopril Trial where patients with diabetic nephropathy randomized to captopril had a tandem decrease in proteinuria and rate of progression of nephropathy compared to those in the placebo arm [16]. This observation was confirmed in the aforementioned RENAAL study because of which it was clear that diabetic nephropathy patients randomized to losartan (versus placebo) achieved a $35 \%$ reduction in proteinuria and were $16 \%$ less likely to reach a renal endpoint [17]. Similarly, the IDNT assigned patients with diabetes and chronic kidney disease to irbesartan, amlodipine, or placebo [18]. Individuals treated with irbesartan experienced a $33 \%$ reduction in proteinuria compared to $10 \%$ among placebo recipients 
and this group had a $20 \%$ reduction in primary renal endpoints.

The African American Study of Kidney Disease and Hypertension (AASK), while conducted exclusively in African American patients without diabetes, is noteworthy given the advanced degree of renal failure (mean creatinine $2.2 \mathrm{mg} / \mathrm{dl}$ ) and high-risk African American population [19]. Study participants received metoprolol, amlodipine, or ramipril with proteinuria declining by similar amounts (15\%) in the metoprolol and ramipril arms. However, despite similar levels of proteinuria reduction, those on ACEi therapy were $22 \%$ less likely to experience a renal endpoint compared to metoprolol-treated patients. In sum, a reduction in proteinuria among diabetic nephropathy patients receiving either ACEi or ARB monotherapy portends improved renal survival and therefore can be used as a surrogate marker for the progression of diabetic kidney disease.

The alternate therapy arms in the aforementioned IDNT and AASK trials further reinforce the observation that reductions in proteinuria are associated with a slowing of nephropathy progression. In the IDNT trial, amlodipine therapy failed to reduce proteinuria by more than $5 \%$ compared to placebo with such patients reaching the primary renal endpoints with equal frequency as placebo. In the AASK trial, patients assigned to amlodipine recorded a $60 \%$ increase in proteinuria over the course of the trial ultimately leading to early termination of the amlodipine arm.

In light of the antiproteinuric effects and slowed nephropathy progression among patients receiving ACEi or ARB monotherapy, subsequent trials attempted to reduce proteinuria further using dual renin-angiotensinaldosterone system (RAAS) blockade. The Ongoing Telmisartan Alone or in Combination with Ramipril Global Endpoint Trial (ONTARGET) was the first large trial to compare ramipril to ramipril plus telmisartan on cardiovascular events among those with diabetes or vascular disease [20]. A similar numbers of patients in each arm reached the cardiovascular endpoints; however, the incidence of hyperkalemia, hypotension, and 'renal impairment' was significantly more common in those assigned to combination therapy. Thereafter, the Aliskerin Trial in Type 2 Diabetes Using Cardiorenal Endpoints (ALTITUDE) Trial evaluated dual therapy on cardiovascular and renal events [21]. The trial was prematurely terminated because while proteinuria reduction was $14 \%$ greater among those patients on combination therapy, these participants experienced significantly more hyperkalemia and hypotension. Finally, worse cardio- vascular outcomes were observed as a trend in those on dual therapy.

The most recent trial, the Veterans Affairs Nephropathy in Diabetes (VA NEPHRON-D), specifically evaluated the effects of combination therapy with lisinopril and losartan on renal outcomes such as the glomerular filtration rate (GFR) and progression to ESRD [22]. The trial was halted early because, despite improvement in proteinuria, patients randomized to ARB plus ACEi therapy experienced an increased risk of hyperkalemia and acute kidney injury. Also a trend toward worse renal outcomes was observed in patients when compared to the outcomes in placebo-treated patients.

Taken together, the data indicate that a blood pressure goal of less than 140/90 mm Hg can optimally slow CKD progression. Blood pressure levels of less than $130 / 80 \mathrm{~mm}$ $\mathrm{Hg}$ are indicated in those with an estimated GFR of less than 60 and more than $500 \mathrm{mg}$ of urinary protein, although the evidence is based exclusively on retrospective analysis and is weaker than the $140 / 90 \mathrm{~mm} \mathrm{Hg}$ goal. ACEi or ARB therapy is compulsory for those with $>300 \mathrm{mg}$ of albuminuria and an estimated GFR of less than $60 \mathrm{ml} /$ $\mathrm{min}$. Otherwise, there is little in the way of evidencebased guidelines for specific agents, particularly in early $\mathrm{CKD}$, where the focus is on blood pressure control, regardless of the prescribed agent. Finally, dual RAAS blocking therapy is contraindicated in all populations, since it increases the risk for hyperkalemia, vulnerability to acute kidney injury, and may increase the risk for allcause mortality.

\section{Disclosure Statement}

Dr. Sternlicht has no conflicts of Interest. Bayer.

Dr. Bakris receives grant support from Relypsa, Medtronic and

$\mathrm{He}$ is a consultant for AbbVie, Bayer, Boeringher-Ingelheim, Takeda, NxStage. He is the Editor of American Journal of Nephrology and Nephrology/Hypertension section of UpToDate and Associate Editor of Diabetes Care, Hypertension Research and Nephrology, Dialysis and Transplantation.

References

1 Epstein M, Sowers JR: Diabetes mellitus and hypertension. Hypertension 1992;19:403-418.

2 Nørgaard K, Feldt-Rasmussen B, Borch-Johnsen K, Saelan H, Deckert T: Prevalence of hypertension in type 1 (insulin-dependent) diabetes mellitus. Diabetologia 1990;33:407-410.

3 Yamout H, Lazich I, Bakris GL: Blood pressure, hypertension, RAAS blockade, and drug therapy in diabetic kidney disease. Adv Chronic Kidney Dis 2014;21:281-286. 
4 Tight blood pressure control and risk of macrovascular and microvascular complications in type 2 diabetes: UKPDS 38 . UK prospective diabetes study group. BMJ 1998;317:703-713.

5 Patel A, MacMahon S, Chalmers J, et al: Effects of a fixed combination of perindopril and indapamide on macrovascular and microvascular outcomes in patients with type 2 diabetes mellitus (the ADVANCE trial): a randomised controlled trial. Lancet 2007;370:829-840.

6 Jamerson K, Weber MA, Bakris GL, et al: Benazepril plus amlodipine or hydrochlorothiazide for hypertension in high-risk patients. N Engl J Med 2008;359:2417-2428.

7 Emdin CA, Rahimi K, Neal B, Callender T, Perkovic V, Patel A: Blood pressure lowering in type 2 diabetes: a systematic review and meta-analysis. JAMA 2015;313:603-615.

8 Cooper-DeHoff RM, Gong Y, Handberg EM, et al: Tight blood pressure control and cardiovascular outcomes among hypertensive patients with diabetes and coronary artery disease. JAMA 2010;304:61-68.

9 Cushman WC, Evans GW, Byington RP, et al: Effects of intensive blood-pressure control in type 2 diabetes mellitus. N Engl J Med 2010; 362:1575-1585.

10 Anderson RJ, Bahn GD, Moritz TE, et al: Blood pressure and cardiovascular disease risk in the veterans affairs diabetes trial. Diabetes Care 2011;34:34-38.
11 Taler SJ, Agarwal R, Bakris GL, et al: KDOQI US commentary on the 2012 KDIGO clinical practice guideline for management of blood pressure in CKD. Am J Kidney Dis 2013;62: 201-213.

12 Weber MA, Schiffrin EL, White WB, et al: Clinical practice guidelines for the management of hypertension in the community a statement by the American society of hypertension and the international society of hypertension. J Hypertens 2014;32:3-15.

13 Perry HM Jr, Miller JP, Fornoff JR, et al: Early predictors of 15-year end-stage renal disease in hypertensive patients. Hypertension 1995; 25:587-594.

14 Bakris GL, Weir MR, Shanifar S, et al: Effects of blood pressure level on progression of diabetic nephropathy: results from the RENAAL study. Arch Intern Med 2003;163: 1555-1565.

15 Pohl MA, Blumenthal S, Cordonnier DJ, et al: Independent and additive impact of blood pressure control and angiotensin II receptor blockade on renal outcomes in the irbesartan diabetic nephropathy trial: clinical implications and limitations. J Am Soc Nephrol 2005; 16:3027-3037.
16 Lewis EJ, Hunsicker LG, Bain RP, Rohde RD The effect of angiotensin-converting-enzyme inhibition on diabetic nephropathy. The collaborative study group. N Engl J Med 1993; 329:1456-1462.

17 Brenner BM, Cooper ME, de Zeeuw D, et al: Effects of losartan on renal and cardiovascular outcomes in patients with type 2 diabetes and nephropathy. N Engl J Med 2001;345:861-869.

18 Lewis EJ, Hunsicker LG, Clarke WR, et al Renoprotective effect of the angiotensin-receptor antagonist irbesartan in patients with nephropathy due to type 2 diabetes. $\mathrm{N}$ Engl J Med 2001;345:851-860.

19 Wright JT Jr, Bakris G, Greene T, et al: Effect of blood pressure lowering and antihypertensive drug class on progression of hypertensive kidney disease: results from the AASK trial. JAMA 2002;288:2421-2431.

20 Yusuf S, Teo KK, Pogue J, et al: Telmisartan, ramipril, or both in patients at high risk for vascular events. N Engl J Med 2008;358:15471559.

21 Parving HH, Brenner BM, McMurray JJ, et al: Cardiorenal end points in a trial of aliskiren for type 2 diabetes. N Engl J Med 2012;367: 2204-2213.

22 Fried LF, Emanuele N, Zhang JH, et al: Combined angiotensin inhibition for the treatment of diabetic nephropathy. N Engl J Med 2013;369:1892-1903. 